\title{
La relación entre el equipo de salud y la familia del niño muriente
}

\section{Mara Muñoz Salinas ${ }^{1}$}

Resumen: La presente es una revisión basada en búsqueda bibliográfica, realización de entrevistas y en la experiencia de la autora acerca del proceso de atención, tal como se realiza en la actualidad, de aquellos padres que enfrentan la muerte de un hijo. La investigación se enmarcó dentro del paradigma cualitativo, usando la entrevista semiestructurada como método de recolección de datos. Se obtuvo una serie de respuestas en las que se observa la percepción de los padres acerca de los cuidados del equipo de salud, principalmente de los profesionales de enfermería, desde el diagnóstico hasta el momento de duelo en que ellos se encuentran.

Palabras clave: duelo, pérdida, relación clínica, bioética

\section{The relation between health care team and the family of dying child}

\begin{abstract}
The present study is a review based on bibliographic search, interviews and the experience of the author about health care process, as it is currently done, for parents that face the death of their child. This research belongs to the qualitative paradigm, using a semi structure interview as method of data recollection. Several answers were obtained seeing parents perception about health care of health team, mainly of nursing professionals, from diagnosis to the moment of mourning they are.
\end{abstract}

Key words: mourning, loss, clinical relation, bioethics

\section{A relação entre a equipe de saúde e a família da criança moribunda}

Resumo: A presente é uma revisão baseada na busca bibliográfica, na realização de entrevistas e na experiência da autora sobre o processo de atenção, tal como se realiza na atualidade, daqueles pais que enfrentam a morte de um filho. A pesquisa teve como marco o paradigma qualitativo, usando a entrevista semi-estruturada como método de coleta de dados. Foi obtida uma série de respostas nas quais se observa a percepçáo dos pais sobre os cuidados da equipe de saúde, principalmente dos profissionais de enfermagem, desde o diagnóstico até o momento de enfrentamento em que eles se encontram.

Palavras-chave: enfrentamento, perda, relação clínica, bioética

\footnotetext{
${ }^{1}$ Enfermera, Especialista en Pediatría. Profesora de Bioética, Universidad Diego Portales, Chile

Correspondencia: mareva2@gmail.com
} 


\section{Introducción ${ }^{2}$}

El sufrimiento de la familia del niño paciente terminal comienza en el momento mismo del diagnóstico de tal condición. Al observar la ejecución de los roles del equipo de salud intrahospitalario suele encontrarse a los profesionales absortos en múltiples procedimientos o labores administrativas, por lo que el tiempo que destinan a la interacción con los pacientes es percibido por estos como inferior al que efectivamente necesitarían destinar a tal actividad(1).

Frente a un hecho devastador como la cercanía de la muerte de un hijo, la familia del menor sufre un periodo de desajuste que se expande desde el momento del diagnóstico de la enfermedad hasta más allá de la muerte del menor, con procesos de duelo para cuya resolución puede ser de crucial importancia la cercanía y tipo de relación que establezca con quienes son los "detentores de la salud” del que aman. ¿Qué significado otorgan los padres que han sufrido la pérdida de un hijo a la comunicación pre y post muerte con el equipo asistencial?

\section{Antecedentes teóricos y empíricos}

Enfrentarnos a la propia muerte es un movimiento corporal y psíquico que exige la aceptación del concepto de finitud: es el propio cuerpo el sufriente, la vida propia la que acaba. Esto, señala Alizalde(2), es en cierto modo "inelaborable y parcialmente realizable", ya que toca aspectos que nos negaremos a enfrentar, más allá de la destrucción del cuerpo, de la pérdida de las relaciones, de enfrentar la fortaleza o inexistencia de nuestras creencias y trascendencia. Si bien la muerte accidental obvia pasar por esta etapa, la mayoría de las personas debe conocer el enfrentarse al propio fin. Y la seguridad de compañía en el momento extremo es para muchos de ellos, junto al no tener dolor, una necesidad prioritaria.

Cuando esta pérdida se refiere a alguien muy cercano, a alguien tan amado como un hijo, no solo su vida es la que ha quedado trunca, sino que la base misma de la vida de los padres tambalea,

${ }^{2}$ Resumen de Tesis de Magíster en Bioética defendida en enero de 2008, Pontificia Universidad Católica de Chile. Director de tesis: Francisco J. León Correa. dejando en ellos muchas veces una sensación de pérdida de sentido de la existencia y un dolor profundo de superar.

Entender los efectos de un evento tan devastador como este es necesario en el área de la salud, especialmente en el área de la pediatría, en la que se realizan los cuidados comprendiendo como objeto de ellos no solo al paciente nińo, sino que, y en especial cuando se trata de pacientes portadores de patologías graves y prolongadas, a su grupo familiar(3).

\section{El duelo}

Para quien muere, el proceso previo — desde el diagnóstico de su enfermedad (si se lo han explicado) o desde que adquiere la certeza de su muerte inminente - puede ser experimentado de distintas maneras, con un tiempo variable de acuerdo a lo precoz de su diagnóstico y a la capacidad de comprensión de su realidad.

A la vez, quienes son sus cercanos también experimentan el duelo por la pérdida; y para ello cuentan con un tiempo variable que puede facilitar o no el proceso completo, ya que pueden acentuarse culpas y estados de desolación que retardan un proceso de adaptación.

Cuando a alguno de nuestros seres queridos se le diagnostica una enfermedad terminal se despiertan en nosotros profundos miedos y tristezas, $y$ experimentamos la pérdida de seguridad de nuestro mundo: debemos prepararnos para lo que vendrá. Hablamos aquí del duelo anticipatorio, proceso generalmente asociado con la persona que ha sido diagnosticada, pero que, en efecto, acompaña también a quienes son su círculo cercano, sobre todo cuando se trata de enfermedades largas.

El duelo anticipatorio es un proceso silencioso, que además no se comenta, en el que quien lo experimenta se centra en el dolor que le espera tras la pérdida. Puede tener una duración variada y ser potenciado por las transiciones de salud y eventual merma en calidad de vida que experimente el muriente. 
Elisabeth Kübler-Ross ha descrito "las cinco etapas del duelo: negación, ira, negociación, depresión y aceptación", ampliamente conocidas. Existen diversos estudios que también han ilustrado el proceso del duelo, identificando a su vez estadios de embotamiento de la sensibilidad, de incredulidad, de desorganización y reorganización. Dichas etapas no constituyen un todo rígido, sino que, tal como han observado oportunamente Bowlby y Saunders(4), quien está frente a una pérdida grave (por ejemplo la propia vida) puede atravesarlas en distinto orden, volviendo a veces a algunas que se creían superadas, o detenerse tiempos prolongados en una de ellas para luego saltarse otras. El duelo es un proceso personal en que cada individuo coloca sus vivencias, temores y culpas, y en el que el proceso de asumir la falta de alguien amado puede no tomar el tiempo que para el observador externo pudiera parecer "normal".

Con esta sucesión de pasos se llega generalmente a la aceptación: hay un reordenamiento, una recomposición de la vida, que puede que no sea la que se quiere, pero es la que se entiende como real y propia, con una carencia dolorosa pero verdadera. Y a mayor conexión con el ser que se ha perdido, más duro y prolongado parece ser este proceso.

De esta forma, gradual y propia, se forma una nueva vida, una diferente, con una aceptación a veces a pedazos, en que se arma una nueva relación con quien ha muerto y se reintegra la vida del doliente con la ausencia de quien se perdió.

En el caso de los padres de niños portadores de una enfermedad terminal, el duelo anticipatorio comienza el día en que se les da el diagnóstico. Pero este duelo anticipatorio es distinto del dolor después del deceso.

De los estudios de Bozeman, de Friedman y de Wolff se obtiene una visión bastante completa de las etapas iniciales del duelo en estas familias, describiendo al inicio una sensación de "aturdimiento", de percepción de irrealidad de todo lo que acontece, necesitando a veces de varios días para asimilar la información. Hay luego una fase de incredulidad de la información médica, cuestionando el diagnóstico. Unida a esta incredulidad puede haber otras manifestaciones emocionales o comportamentales, propias de un proceso adaptativo difícil como éste:

- Enojo contra quienes hacen o aceptan el diagnóstico que se ha entregado, en especial con el equipo de salud.

- Accesos de actividad intensa, cuidados ansiosos otorgados al hijo, búsqueda frenética de información sobre la enfermedad.

- Culpa: se acusan a sí mismos de no haber pesquisado a tiempo la enfermedad, de no haber llevado oportunamente al médico, de haber portado un gen defectuoso; si se es religioso, puede haber percepción de la enfermedad del hijo como la falta a un precepto divino.

- Problemas con la información: pueden suceder frecuentes desacuerdos entre los padres sobre cuándo informar al enfermo o a sus hermanos del estado de su mal. Esto lleva a la entrega de datos confusos que no hacen sino minar la confianza entre los miembros de la familia.

Aunque toda esta fase de incredulidad puede durar incluso meses después de la muerte del hijo, Chodoff, ya en 1964, describió cómo, en el caso de niños cuya fase terminal era prolongada, se observaba de parte de los padres una disminución en la carga emocional que depositaban en el hijo; así, al haber una disminución, un debilitamiento de la aflicción, al llegar la muerte se observaba una resignación facilitada por el proceso de duelo anticipado. Las conclusiones de los estudios del NIMH muestran que la forma en que reaccionen los padres a la noticia de la enfermedad del hijo, cómo respondan durante las primeras semanas, determinará en gran medida su patrón comportamental en adelante.

El periodo de cuidados terminales que corresponde temporalmente a estas fases es un tiempo muy importante para los padres, pues les permitirá, en este duelo anticipatorio, prepararse para la pérdida y prodigarle cuidados y cariños que enriquecerán su relación y aliviarán y consolarán al niño.

En este tiempo, puede ser impactante para los padres percatarse no solo de un desfase en cómo se vivencian estos tiempos, sino también en el co- 
nocimiento efectivo que el nińo tiene de su situación. Así, son los niños los que, a menudo, intuyendo o conociendo dentro de sus capacidades cuál es su pronóstico, llegan a la aceptación mucho antes que los padres, vivenciando el proceso terminal en forma más serena que la que ellos esperarían, e incluso consolándolos y preparándolos para el momento de la separación. Esto último, si bien en un primer momento puede descolocar a la familia, luego es recordado como un consuelo y una ayuda. A la vez, para el niño moribundo no hay mejor consuelo que la presencia y seguridad de sus padres.

En el alto grado de distorsiones en la realidad familiar, es de alta importancia considerar la convulsión en el proceso de desarrollo de los hermanos del niño muriente. Más aún si se toma en cuenta lo frecuente del hacerlos "responsables" del sufrimiento del enfermo, tendencia por demás dañina aunque inconsciente, y que se hace más frecuente cuando la muerte es repentina.

Que no se explique a los hermanos del niño enfermo lo que ocurre tan claramente como sus edades lo permiten puede generar un cúmulo de fantasías terroríficas que expliquen los llantos y ansiedades del resto de la familia, la falta de expresiones de cariño y cuidado, el sufrimiento y la ausencia de quien fallece. Esto, además de proporcionarles una carga extraordinariamente negativa para el enfrentamiento de pérdidas y de la misma muerte, puede dejar una carga de culpas y temores que se expresen en conductas desadaptativas en su posterior desarrollo.

Del mismo modo, que se les permita participar de los cuidados o simplemente acompañar al hermano enfermo, darles un momento para despedirse y participar del funeral, en la medida en que ellos quieran, facilita un duelo sano, que no dañe el desarrollo de la personalidad de los nińos.

\section{Acompańamiento en fase terminal y pérdida}

Tras haber descrito investigaciones y experiencias acerca del enfrentamiento de niños y familias a la pérdida y la muerte, se vuelve importante observar el planteamiento realizado por Doka (1993)(5) de un modelo de cuidados frente a quien experimenta una enfermedad terminal.

Plantear el enfrentamiento a estos procesos de las personas bajo nuestro cuidado es útil en cuanto permite proyectar objetivos a lograr en cada etapa en que se encuentren. Corr \& Corr, basándose en los estudios de Doka, Weisman (1980) y Pattison (1969, 1978), afirman:

- En la fase de prediagnóstico, cuando aún no está claro sino el inicio de una enfermedad y muchos exámenes, el niño y sus padres presentan distintos niveles de miedo y culpas, a la vez que gran necesidad de información sobre lo que ocurre. Sería entonces necesario enfocarse en entregar no sólo información acerca de los posibles diagnósticos, sino también averiguar cuáles son sus temores y cómo los enfrentan, de modo de evitar que luego se presenten culpas.

- En la fase aguda, centrada alrededor de la crisis que es un diagnóstico de riesgo vital, muchas familias han relatado estrés extremo, a un punto que solo es superado por el trauma de la pérdida, marcando para ellos claramente una línea divisoria entre dos distintos estilos de vida: uno en el que veían el porvenir como algo seguro y optimista; el otro en el cual todo es temible, potencialmente peligroso y totalmente incierto. Se percibe prácticamente un secuestro de lo que fue la vida familiar. Se debe establecer aquí la utilización del soporte social que, a estas alturas, aún dan útilmente familia y amigos, y promover planificaciones a corto plazo, ya que la familia se siente incapaz de percibir más allá en su futuro.

- En la fase crónica, caracterizada por el seguimiento de múltiples terapias, la familia se enfrenta a una lucha por la vida, mientras existen una serie de problemas derivados de la enfermedad y su tratamiento. Todo esto podría comprometer la capacidad del pequeño y su grupo familiar de lograr la adecuación a las demandas que ahora existen. Junto a esto, sus oportunidades de socializar se ven disminuidas por los tiempos de tratamiento, y muchas familias cercanas podrían sentir poco grato la interacción con ellos en estas circunstancias. Los cuidados, entonces, además de privilegiar la terapia y la prevención de sus efectos colaterales, deben considerar estimular y asegurar 
la adherencia de la unidad niño-familia al tratamiento, más allá de todas estas incomodidades, y potenciar que se enfoquen como grupo en lograr la mejor adaptación del niño a los cambios que experimenta.

- Fase terminal: en esta etapa, en general, los objetivos se condicen con los cuidados paliativos: minimización de causas de estrés y maximización de calidad de vida, además de la preparación del grupo familiar para la muerte. Frecuentemente, la familia tarda un tiempo en aceptar esta nueva orientación de los cuidados, ya no luchando por la vida, sino iniciando un camino de separación.

- Fase de recuperación: no todas las enfermedades con riesgo vital terminan en muerte. Sin embargo, debe considerarse también el apoyo para el niño superviviente y su familia, ya que haber pasado por una experiencia extrema, como es la lucha por la vida, dejará en él una profunda marca, más allá de las secuelas físicas que puedan haber quedado, y la percepción de inseguridad y temor persistirán mucho tiempo, debiendo fortalecer los aspectos emocionales y psicológicos para promover un desarrollo saludable.

La experiencia británica, descrita por Colin $\mathrm{Mu}-$ rray y Marilyn Relf en "Counselling in terminal care and bereavement"(6), señala que, en general, un escaso porcentaje de familias requiere un acompañamiento prolongado y esto ocurre casi siempre en la segunda etapa; la primera, si bien caracterizada por eventos que persistirán en la memoria de los sobrevivientes (el fallecimiento, la entrega y preparación del cuerpo, el velatorio, el funeral, el entierro), está también marcada por la presencia constante de familia y amigos. Es un tiempo de ruido y compañía además de un cúmulo de sentimientos en pugna.

Sin embargo, aun así, al equipo de salud, puntualmente a las enfermeras, muchas veces les corresponderá desempeñar roles paternales o maternales, en el sentido de sustituir o reforzar comportamientos, realizar acciones concretas en el momento de ocurrir la muerte, (propiciar una atmósfera adecuada para la despedida, acompañar al familiar mientras le dan a conocer lo ocurrido, incluso la preparación final del cuerpo de quien fuera su paciente), y sugerir acciones a seguir por una familia muchas veces abrumada.

Una presencia cariñosa, los cuidados respetuosos hacia el cuerpo en el primer instante y una figura que más que hacer sepa estar, dando seguridad en esos momentos de incertidumbre máxima, pueden ser vistos como un consuelo real.

En esos instantes puede ofrecerse la presencia de un sacerdote o persona religiosa; incluso en aquellas familias que han declarado no profesar ninguna religión, la presencia de un agente pastoral puede ser vista como necesaria. Esto, además de satisfacer la profunda inquietud espiritual que pueda haber en la familia, les permite la percepción de un nexo más allá de lo momentáneo y doloroso con quien se está cuidando.

En nuestra realidad sanitaria, es inexistente en la fase de ajuste el acompañamiento a la familia de quien murió. Si bien se intuye lo necesario que pueda ser, en general y salvo iniciativas individuales el equipo de salud no vuelve a tomar contacto con la familia, muchas veces por suponer que esto les podría significar un mayor dolor al revivir la pérdida.

En el artículo británico citado se menciona que es en esta segunda fase cuando mayor apoyo se necesita: cuando ya no hay tanto "ruido" y sí se siente la ausencia en forma permanente y la necesidad de recordar aspectos del fallecido con otra persona que quiera escuchar. En el estudio de Murray \& Relf, como en otros aludidos en estas páginas, se afirma que, en la medida en que haya sido posible prepararse para la pérdida e incluso participar en los cuidados a quien moría, podría no ser tan necesaria esta ayuda. Aun así hay casos, concluyen, en que el acompańamiento es necesario, sobre todo en aquellos en que la pérdida ha sido tan grande como la muerte de un hijo.

Dice Haring: "Los profesionales de salud deberemos aprender a ayudar al muriente y a su familia a que vivan su muerte" $(7)^{3}$. La vida e incluso la alegría que el personal que atiende a los pacientes terminales irradia tiene su base en la compasión humana práctica que se realiza, en los cuidados, el manejo de los síntomas, el apoyo psicológico.

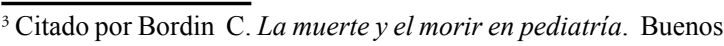
Aires: Lumen; 1997. 
Y esto, producto de una actitud de respeto por el paciente y de una actividad corporativa, en que el individualismo es balanceado por el trabajo en equipo, y esto da paso a un trabajo fluido.

Es necesario destacar la labor del enfermero como un profesional que posee un bagaje de conocimientos especializados y la capacidad para aplicarlos, con el propósito de ayudar a otros seres humanos a encontrar un sentido a la enfermedad o mantener el máximo nivel posible de salud, en una relación persona a persona, de acuerdo con el concepto desarrollado por Joyce Travelbee(8), en el que se consiga un acercamiento empático en periodos de real necesidad y cuestionamiento del sentido de la vida. El enfermero es, en efecto, el profesional que tiene como razón de ser cuidar a la persona en las distintas etapas de la vida. De igual modo, cómo se vea afectado por cada evento de estas características con que se enfrente será relativizado por sus propias pautas de formación y apoyo.

\section{Métodos}

La investigación, necesariamente de tipo retrospectivo, se realizó en el marco del método cualitativo, seleccionando como diseño instrumental la entrevista semiestructurada.

El marco de esta exploración se delimitó entonces sobre la base de las perspectivas del paradigma interpretativo/fenomenológico, en el cual se fundamenta la importancia de la "espacialidad, corporeidad, temporalidad y relacionabilidad”, bases de la realización de esta investigación que involucró las percepciones y las sensaciones de los padres de pacientes en etapa muriente, ya que la obtención y el análisis de datos estuvieron directamente ligados a la realidad de estas personas, describiendo el escenario en el cual se desarrollan los acontecimientos que se investigan. De la misma forma, contempló la identificación de los diversos fenómenos y características de la conciencia y las estructuras mentales de las personas enfrentadas a la muerte del ser amado.

\section{Campo de estudio}

La muestra correspondió a una muestra dirigida, seleccionada por el tiempo de ocurrida la pérdida (entre 3 meses y 4 años).

\section{Credibilidad}

Para contrastar los hallazgos sobre lo referido por los padres se contó con la bibliografía analizada. Así, la triangulación necesaria fue teórica, referida principalmente a los estudios que realizaron Relf y Bowlby.

Una breve visión de la contraparte, la visión de las enfermeras sobre el desempeño de su rol ante paciente muriente y su familia, así como del rol institucional, lo encontramos en la tesis de Escalona y González(9).

Se evitó triangular con procesos de observación directa de procesos relacionados con atención de equipo de salud-paciente, dado que, al ser la mayor parte del estudio realizado en el lugar de labor clínica de la autora, podía haber algún sesgo de error al subjetivizarse la interacción.

\section{Resultados}

En un primer nivel de análisis se exponen los datos obtenidos a través de las entrevistas en profundidad. Se presenta también un segundo nivel de análisis, construido desde la fenomenología y de acuerdo con lo propuesto en la teoría fundamentada, dando cuenta de la elaboración que hacen los padres que han sufrido la pérdida de un hijo del rol que cumple el equipo de salud a través del tiempo.

\section{Análisis de los datos de primer nivel}

Con los datos recogidos a través de notas y grabaciones, la entrevistadora transliteró el discurso de los padres participantes en el estudio, extrayendo a través de lecturas reiteradas los temas emergentes. Sobre esta base se organizaron las matrices expuestas y se efectuó el orden necesario para la realización de la siguiente etapa.

Luego de establecidas las categorías y subcategorías, se observó la forma en que estas se relacionaban.

Descripción de los datos obtenidos a través de las 
entrevistas en profundidad realizadas a padres que han sufrido la pérdida de un hijo. Dimensiones: tiempo, espiritualidad, duelo, relación con equipo de salud.

En esta última dimensión se especifica, más allá de las acciones realizadas por el personal médico y no médico que estuvo en contacto con ellos (no sólo información, terapias y cuidados), cuál es la percepción de la relación que se sostuvo con el equipo de salud debido a los cuidados otorgados al hijo. Esto a través de las siguientes categorías: percepción de agresión, percepción de abandono, apoyo, cercanía.

\section{Relación de categorías: entrevistas a padres que han sufrido la pérdida de un hijo.}

Tal como se denota en el mapa conceptual que sigue, se observa al analizar las entrevistas:

- Los padres tienden a hacer un recuento del tiempo, señalando inconscientemente que para ellos hay un "antes", en que visualizan al hijo sano, asociando a él una serie de buenos conceptos ("luminosa, como guagua parecía iluminada", "El R. fue un gallo espectacular, completo"), y un "después" que se inicia con el diagnóstico de enfermedad.

- El proceso que se inicia entonces, en que ellos como familia enfrentan el proceso de pérdida de la salud y vida del hijo, se ve fuertemente cruzado por la relación con el equipo sanitario ("Era bien regalona de algunas, sobre todo de las auxiliares", "Recuerdo con cariño a muchas tías, a la enfermera que llegaba a explicarme lo que me había dicho el médico antes en palabras que yo no entendía, y a la enfermera que me consolaba y me acompañaba hasta hacerme dormir").

- La relación del equipo de salud con la familia, en la generalidad de los casos observados, desaparece desde el momento en que el niño egresa del establecimiento con diagnóstico terminal, o propiamente en su deceso ("iNunca!, nadie fue, no digo a dar una explicación, pero por lo menos a verlo, a preguntar cómo estaba, y eso que estaban al lado!").

- Se toma nota del elemento religioso como apoyo tangencial de los padres, no observándose una relación del equipo de salud con este tipo de asistencia. Esto toma cierta relevancia cuando se piensa en el centro asistencial en que se realiza el estudio como uno supuestamente ligado a la Iglesia Católica (“¿Dios? A veces le pregunto cosas. Estuve mucho tiempo sin creer en Él", "Ahora sé que tengo una razón para ese día: que cuando yo me muera, mi hijo me estará esperando, que lo voy a encontrar otra vez").

\section{Análisis secundario de datos}

De las observaciones obtenidas se extrajeron categorías centrales (temporalidad, relación familia-equipo de salud, proceso de duelo) que fueron expuestas a la luz de la ética personalista como teoría social, generando conclusiones acerca del desempeño de los roles profesionales e institucionales en la relación con su paciente.

\section{Relación entre temporalidad y duelo}

Entendiendo el duelo como un proceso luego de una pérdida, y considerando que la muerte de un hijo se señala transversalmente como una de las experiencias que causa mayor quebranto, se obtuvo de los padres entrevistados el relato de su vivencia.

En las narraciones se observan las distintas etapas, desde el aturdimiento y desesperación iniciales hasta, en algunos casos, un ajuste hacia esta vida distinta sin el hijo. Una y otra vez se aprecia cómo se fueron superponiendo sentimientos que los padres reflejan claramente en su "antes", cuando disfrutaban a su hijo sano, el desconcierto y miedo inicial frente al diagnóstico, claramente el desarrollo de duelo anticipatorio y, luego, el vacío por la pérdida.

De acuerdo con lo señalado por los estudios de Bowlby y Kübler-Ross, cómo se desarrolle inicialmente este proceso determinará en gran parte el curso del duelo en general. Se observa coincidencia en este punto al notar que, en los casos en que se percibe la acción del equipo de salud como una agresión (reconocida o no esta percepción), hay un desarrollo más prolongado y tórpido del duelo. Del mismo modo, en los casos entrevistados, cuando se logró establecer una relación de ayuda 
adecuada, se observó un mejor y más abreviado encauzamiento hacia el ajuste.

\section{Relación equipo de salud y núcleo familia-pa- ciente}

Aunque frente a una situación de carencia de salud se supone que se establezca una relación de ayuda entre quienes presentan la falta y el equipo sanitario, de las entrevistas realizadas se pueden extractar dos situaciones puntuales:

La percepción de algunos padres del equipo de salud como agresor más que como agente cuidador. Si bien esto puede relacionarse con que son a veces los encargados de dar una noticia tan mala como es el diagnóstico de cáncer en un hijo, o la proximidad de su muerte (Bowlby compara esto al acto griego de matar al mensajero), hay ocasiones en que los padres se refieren con amargura a equipos en los que ellos no pudieron colocar su confianza, o en que los cuidadores no demostraron suficiente preocupación o cercanía hacia el niño. Esto nos podría llamar la atención hacia las capacidades desarrolladas por el equipo para establecer una adecuada y eficaz relación con la familia y el menor, más allá de lo difícil de la situación o de los problemas técnicos que puedan haber enfrentado.

La prácticamente nula presencia del equipo de salud luego del alta definitiva por terminalidad o deceso del paciente. Efectivamente, y tal como se planteaba en el análisis y esquema anteriores, una vez que el paciente egresa del servicio en que recibía las atenciones es prácticamente nulo el contacto con el equipo de salud que hasta entonces cuidaba al niño, excepto escasas ocasiones en que, a título personal, algún miembro del equipo se comunicó telefónicamente o visitó durante el funeral o poco después a la familia ("Yo sé que no tienen todas las respuestas, pero la mano cerca, la que conoció cómo era mi hija, pudo ser de ayuda en ese tiempo").

En el trabajo de Bowlby, así como en experiencias nacionales, como la tesis de Escalona y González, esto es refrendado, haciéndose notar cómo la mayoría de las veces las enfermeras o médicos que continúan el vínculo lo hacen a través de asistir al funeral como una forma de cerrar un proceso personal.
Los padres muestran en general su extrańeza y agradecimiento ante los escasos contactos que tuvieron con las personas del equipo de salud. En general, denotan de ese modo una sensación de abandono de parte de ellos, reconociendo la necesidad que habrían tenido de conversar sobre lo vivido con alguien "que hubiera conocido" lo que ellos pasaron.

Esto empeora en los casos en que el niño salió para morir en su casa, encontrándose los padres en situaciones de indefensión inesperadas, al no saber cómo responder a las necesidades de sus hijos, e incluso enfrentarse a la imposibilidad de obtener medicamentos para combatir el dolor.

No deja de ser llamativa esta situación si se contrasta con la definición pediátrica que propende una relación terapéutica con la unidad pacientefamilia. Queda claro que, si bien el niño es atendido en la forma más completa que el equipo disponga durante su estadía, y que durante este tiempo la familia es vista como el agente que prolonga los cuidados, informada y hecha parte de los cuidados, una vez se da el alta por terminalidad del niño no hay otro lazo efectivo hacia ella.

Que se presenten estas realidades obviamente provoca el cuestionamiento, ya que el proceso al que esta familia se enfrenta es especialmente duro y delicado, especialmente necesitado. Para ella, no estar inserta en la dinámica del establecimiento significa la ausencia de lazos con un personal calificado para comprender sus necesidades y procesos. Y, si bien es lógico pensar que terminada la relación cliente-institución no cabe otra responsabilidad al respecto, surge también el cuestionamiento a la labor del equipo, desde su compromiso deontológico con la mirada compasiva que debe a su paciente (y si era un binomio cuando estaba bajo su tutela, ¿no queda una parte del binomio en estado de necesidad?).

\section{Conclusión y sugerencias}

Las experiencias difíciles o dolorosas en la vida desencadenan naturalmente repuestas de apego y no es extraño que, enfrentados a alguien que muere, desarrollemos conductas evasivas. Que quien muera sea alguien cercano podría desenca- 
denar entonces diversos problemas, pero podría también enseñar algo sobre separación y pérdida. Así también para quien muere se convierte el proceso, cuando existe una enfermedad terminal prolongada, en una oportunidad de revisión de lo vivido hasta entonces, de preparación para el proceso que llega.

Cuando quien muere es un niño esto no cambia. Diversos observadores han expuesto que todo niño se da cuenta de la cercanía de la muerte. Sin embargo, el tabú del que hemos rodeado a la muerte ha hecho reforzar una y otra vez la creencia de lo poco adecuado que es hablar del tema con los pequeños, incluso cuando son estos quienes están en proceso de muerte.

Doctores, enfermeras $\mathrm{u}$ otros profesionales no sustituyen la presencia de la familia, pero pueden ayudar a la familia real a acercarse a "la línea de fuego", y si ellos desarrollan actitudes disfuncionales, es posible ayudarlos a descubrir distintas formas de encarar la situación y romper un círculo vicioso que podría perpetuar los problemas. La formación de equipos que acompañen en forma adecuada a estas familias se hace necesaria, desde que son ellas quienes acuden al equipo de salud en busca de ayuda y desde que, sobre todo en pediatría, se asume que los cuidados deben siempre considerar no sólo al niño sino al binomio niñofamilia.

Enriquecer el conocimiento en esta área sería de importancia crucial para enfrentar procesos que son dolorosos y para los cuales no existen en realidad redes de apoyo específicas y efectivas en salud, para prestar ayuda al paciente y su familia en un proceso trascendental y, finalmente, para cumplir con el acompañar al paciente hacia un bien morir.

En el mundo de la atención de salud, cuando el paciente, a pesar de todos los esfuerzos, técnicas y tecnologías, empeora, aun cuando la conciencia y conocimiento profesional digan lo contrario, puede percibirse esto como un fracaso. Y esta percepción de fracaso, unida a los sentimientos que podamos vivenciar por el paciente (no, no es un cliente), pueden coludirse para permitir su abandono y el de su familia. Y aún nuestro sistema no concibe espacios efectivos para quienes sufren la pérdida.

Sin embargo, sería necesario asumir otro rol. Aparecen aquí múltiples posibilidades de asistencia y sugerencias de formación de equipos de apoyo multiprofesional desde la perspectiva paliativista. Otros, orientados al paciente en estado crítico, han llegado a formular guías de atención a familiares(10) que sería prudente revisar en vistas a idear marcos de comunicación efectiva. No obstante, sobre la base de la revisión exhibida y dadas las características de los pacientes que se observaron en esta ocasión, se proponen las siguientes sugerencias de intervención, coherentes además con los Principios de Terapéutica y de Sociabilidad planteados por Mons. Elio Sgreccia(11):

Desde una profunda reflexión sobre la práctica clínica fundada en la compasión del sufrimiento del otro(12), se hace necesario plantear una propuesta educativa orientada a capacitar efectivamente y ya en el pregrado al equipo de salud en temas de cuidados paliativos y en tanatología, para obtener mayor conocimiento acerca de las necesidades que los pacientes, sus familias, y el equipo mismo presentarán, y cuáles serían las herramientas para satisfacerlas. Y esto subrayando la necesidad de establecer reales relaciones de ayuda, en que todo el equipo de salud conozca los deseos de cada familia, para lograr un proceso de despedida y duelo en la forma más natural.

Estimular la estructuración de réplicas de la experiencia inglesa relatada por Relf, en cuanto a la formación de monitores que realicen acompañamiento telefónico, durante un tiempo a delimitar, a aquellas familias que enfrenten situaciones de pérdida; esto en el espíritu de la misión/visión de la institución, en su compromiso con el bienestar de la persona humana y con la mirada en una labor de promoción de salud mental efectiva de la comunidad ligada a la institución. 


\section{Referencias}

1. Gutiérrez P, Núnez E, Rivera C. Características del rol en el profesional de enfermería intrahospitalario. Rev Enf Chile 2002; 120: 22-28.

2. Alizalde AM. Clinicas con la muerte. Buenos Aires; 1995: cap. 5.

3. Whaley \& Wong. Enfermería pediátrica. Tomo I. Barcelona: Mosby-Doyma; 1995: 9.

4. Saunders C. Cuidados en la enfermedad maligna terminal. Barcelona: Salvat; 1988.

5. Corr \& Corr. Handbook of childhood death and bereavement. New York: Springer Publishing Company; 1997: 90.

6. Murray C, Relf M, Couldrick A. Counselling in terminal care and bereavement. Canterbury: BPS Book; 1997.

7. Bordin C. La muerte y el morir en pediatría. Buenos Aires: Lumen; 1997.

8. Marriner A. Modelos y teorias en enfermería. Madrid: Elsevier; 2007.

9. Escalona C, González V. Enfrentamiento de la muerte de un paciente desde el punto de vista de la enfermera. Santiago de Chile: Universidad Diego Portales; 2006.

10. García Mozo A, Sánchez Roldán F, Amorós Cerdá SM, Balaguer Albarracín F, Diez C, Zaforteza Lallemand

C. Desarrollo de una guía de atención a los familiares del paciente crítico. Enfermería Intensiva 2010; 21: 20-27.

11. Sgreccia E. Manual de Bioética. México D.F.: Diana; 2003.

12. Torralba Roselló F. Constructos éticos del cuidar. Enfermería Intensiva 2000; 11(3): 136-142.

Recibido: 13 de octubre de 2010

Aceptado: 13 de diciembre de 2010 